\title{
Safety Assessment of Vehicles with Ensemble FLC Based FCAS
}

\author{
S. Paul Sathiyan ${ }^{\# 1}$, S. Suresh Kumar ${ }^{\# 2}$, A. Immanuel Selvakumar ${ }^{\# 3}$ \\ ${ }^{\# 1,3}$ Department of Electrical Technology, Karunya University, Coimbatore India \\ ${ }^{1}$ sathiyanme@yahoo.co.in \\ \# 2 Department of Electronics and Communication Engineering, \\ Dr. NGP Institute of Technology, Coimbatore, India
}

\begin{abstract}
This research work proposes a new Ensemble Fuzzy Logic Controller for Forward Collision Avoidance System (EFLC-FCAS) which assists driving in rural, inter urban and highway operating zones. This system is intended to sense the obstacle / lead vehicle in the same lane of travel and apply brake automatically to reduce the potential of rear end collision thus ensuring safety. Article also covers the assessment procedure developed for assessing FCAS. Driver assistance virtual simulation software, CarMaker along with Matlab / Simulink is used for validating the performance of the proposed controller. Test results have shown a positive influence on the performance of the proposed controller for the test procedures recommended by the assessing agencies.
\end{abstract}

Keyword-Forward collision avoidance system, Rear end collision, Autonomous emergency braking assessment, Fuzzy logic controller, CarMaker

\section{INTRODUCTION}

Road transportation plays a very vital role in a country's economic growth. Several driver assistance systems were developed to provide support to the driver and safety to the vehicle occupants by reducing / avoiding the potential of collision. These systems are broadly classified into active and passive safety systems. Active safety system comes into action before the occurrence of any accident. Furthermore this system can be classified into collision warning and collision avoidance systems. In collision warning system, the potential of collision is sensed with the help of LADAR, RADAR etc., and an acoustic / visual warning is issued to the driver. Thereafter the driver needs to take up the control of the vehicle. In collision avoidance system, the controller comes into action on behalf of the driver by applying brakes with the help of Automatic Emergency Braking functionality (AEB) to reduce the speed of the vehicle whereby reducing the potential of the collision. The following figure (Fig. 1) provides the classification of various driver assistance systems.

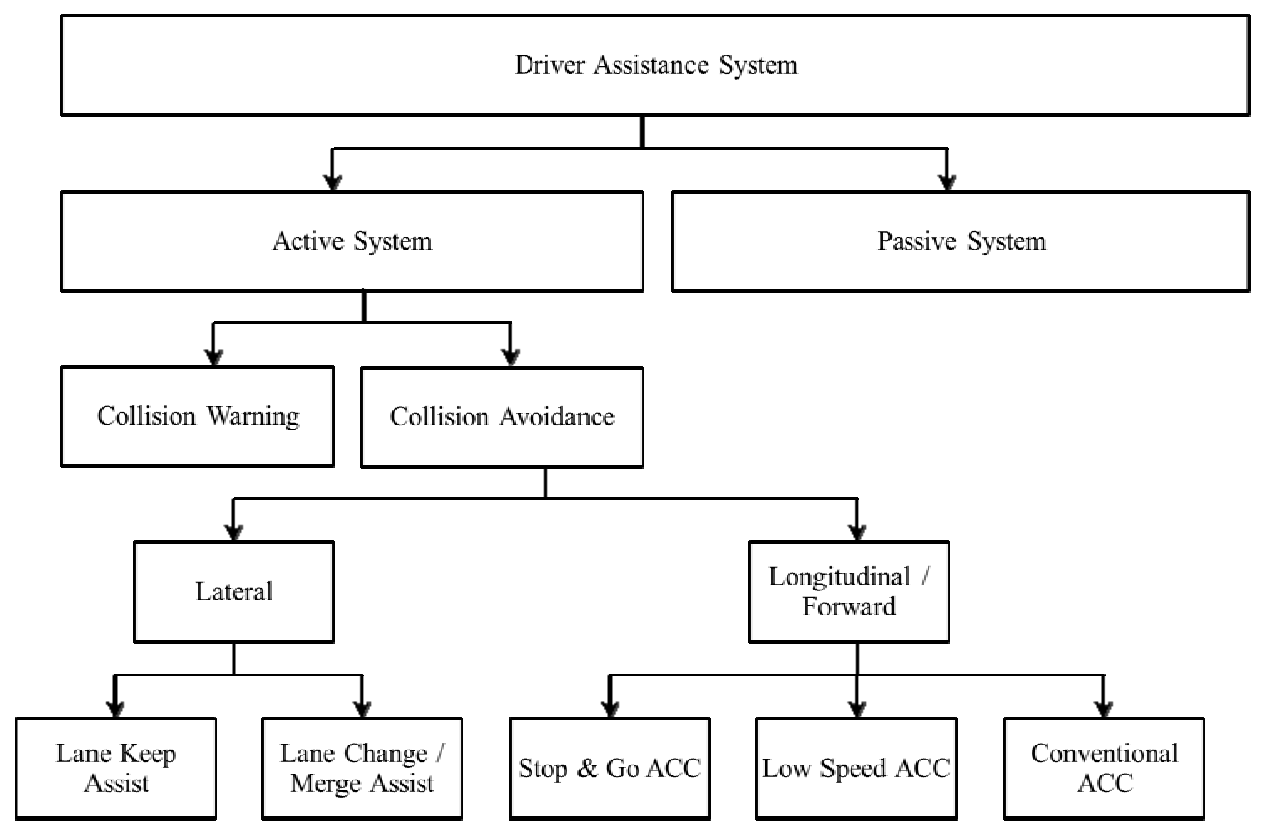

Fig. 1. Driver assistance system classification 
Based on the potential collision risk direction, the collision system can be further classified into lateral and longitudinal / Forward Collision Avoidance System (FCAS) / Vehicle longitudinal control (VLC). Lateral collision avoidance system assists the driver during lane change, lane keeping and merging by sensing the dark spot area. FCAS works by sensing the vehicle / obstacle in front of the subject vehicle (vehicle fitted with FCAS) travelling in the same lane. FCAS can be further classified into Conventional Adaptive Cruise Control (ACC), Low Speed ACC and Stop \& Go ACC system. Each of these classification have their unique characteristics in terms of operating speed, braking efficiency, acceleration and deceleration characteristics. Their operations are bounded to operating zones as defined in Table I.

TABLE I. CLASSIFICATION OF FCAS

\begin{tabular}{|l|l|l|}
\hline \multicolumn{1}{|c|}{ Type } & \multicolumn{1}{c|}{ Operating Velocity Range } & \multicolumn{1}{c|}{ Operating Zone } \\
\hline Conventional ACC & Above $8.33 \mathrm{~m} / \mathrm{sec}$ & Highway \\
\hline Low Speed ACC & Between $1.38 \mathrm{~m} / \mathrm{sec}$ and $8.33 \mathrm{~m} / \mathrm{sec}$ & Rural \\
\hline Stop \& GO ACC & Below $8.33 \mathrm{~m} / \mathrm{sec}$ till 0 & Urban \\
\hline
\end{tabular}

Braking efficiency depends on the vehicle speed at which the brake is applied to bring the vehicle to rest. Braking efficiency is measured in terms of braking distance (distance measured from the point at which the brake is applied to the point at which the vehicle comes into control /stops), clearance distance (distance measured between the obstacle / lead vehicle and the subject vehicle) and the braking duration (time duration between the application of brake and the time when the subject vehicle comes under control / rest). This research work investigates on the braking efficiency of the proposed Ensemble Fuzzy Logic based FCAS (EFLC-FCAS) in all operating zones in comparison with the conventional Fuzzy controllers mentioned in [1] and [2]. Model in loop testing is performed using CarMaker software along with Matlab/Simulink to validate the performance of the proposed controller. The rest of this research article is organised as follows: In section 2, the proposed EFLC-FCAS is discussed in elaborate and the test procedures framed for assessing the performance of the controller is discussed in section 3. Test results are deliberated in section 4 followed by conclusion.

\section{EFLC - FCAS}

In the proposed EFLC-FCAS technique (Fig. 2), two Fuzzy Logic Controllers (FLC) are connected in parallel (FLC 1 and FLC 2). Both these controllers receive the inputs (Velocity Error, $V_{e}$ and Distance Error, $D_{e}$ ) simultaneously through a mux and provide simultaneous control output (throttle / brake, $t / b$ ). Both these errors are the difference between the expected value and the actual value. In order to avoid the rear end collision, these errors must be maintained minimum (theoretically zero). Suitably adjusting the throttle and brake of the vehicle these errors can be maintained minimum. Since FLC is used as the controller, the throttle and brake values are determined by the rule base, the membership function range etc.

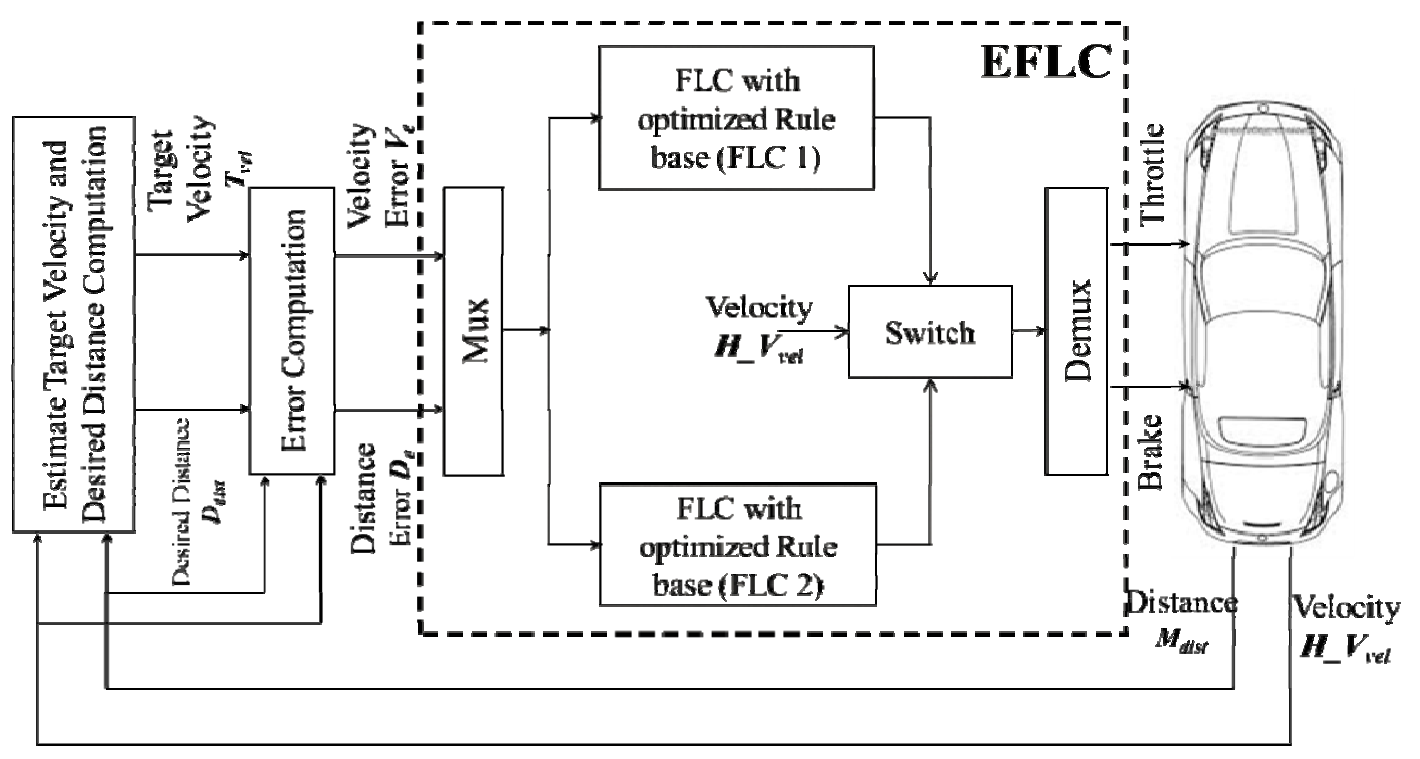

Fig. 2. Proposed EFLC - FCAS architecture 


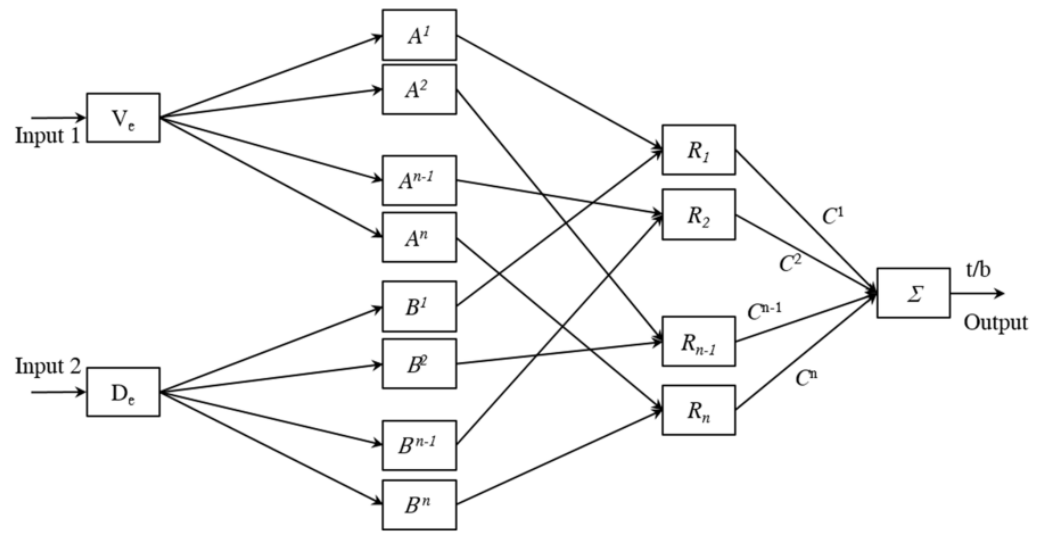

Fig.3. FLC rule formation

The rule base for FLC is formulated with respect to Fig. 3 as follows:

Rule $R_{1}$ : IF $V_{e}$ is $A^{1}$ AND $D_{e}$ is $B^{1}$ THEN $t / b$ is $C^{1}$

Rule $R_{2}$ : IF $V_{e}$ is $A^{n-1}$ AND $D_{e}$ is $B^{n-1}$ THEN $t / b$ is $C^{2}$

Rule $R_{u}$ : IF $V_{e}$ is $A^{n}$ AND $D_{e}$ is $B^{n}$ THEN $t / b$ is $C^{n}$

In general Rule: IF antecendent1 AND antecedent 2 THEN consequence

Where $R$ represent the rule, $u$ represent the number of rules, $A$ is the antecedent of $V_{e}, B$ is the antecedent of $D_{e}$ and $C$ is the consequence. As mentioned in Table II, the ranges of the input and output variables are divided into 9 sub ranges. Hence the total number of possible rules would be $9 * 9 * 9=729$.

The design parameters of the proposed EFLC-FCAS is given in Table II and III. Only one control output will be selected at any point of time (throttle / brake 1 or throttle / brake 2) and used for controlling the vehicle. Following equation helps in understanding the operation of the controller selection.

$$
\text { throttle } / \text { brake }= \begin{cases}\text { FLC } 1, \text { throttle } / \text { brake } 1, & \text { if } \mathrm{H}_{-} \mathrm{V}_{\text {vel }}>V_{\text {thershold }} \\ \mathrm{FLC} 2, \text { throttle } / \mathrm{brake}^{2,} & \text { if } \mathrm{H}_{-} \mathrm{V}_{\text {vel }}<V_{\text {thershold }}\end{cases}
$$

When the subject / host vehicle velocity $\left(H_{-} V_{\text {vel }}\right)$ is above a threshold value FLC 1 will be selected and if the subject vehicle velocity is below the threshold value FLC 2 will be selected. FLC 1 is loaded with the rule base shown in Table IV and FLC 2 is loaded with the rule base shown in Table V. The rule base used in FLC 1 and FLC 2 are developed with an intent to provide the vehicle occupant safety by avoiding rear end collision. These rule bases are formulated using Particle Swarm Optimisation (PSO) and Differential Evolution (DE) respectively [3] . Several testings were performed on the controller which were discussed in [3]-[5].

TABLE II. DESIGN PARAMETERS FOR EFLC-FCAS

\begin{tabular}{|c|c|c|c|c|c|}
\hline & \multirow{2}{*}{ Variables } & \multirow{2}{*}{ Range } & \multicolumn{3}{|c|}{ Linguistic Values } \\
\hline & & & Number & Shape & Names \\
\hline \multirow{2}{*}{ Input } & Velocity Error $\left(V_{e}\right)$ & $\pm 38 \mathrm{~m} / \mathrm{sec}$ & \multirow[b]{3}{*}{9} & \multirow[b]{3}{*}{ Triangular } & \multirow{3}{*}{$\begin{array}{l}\text { Negative Very Large } \\
\text { Negative Large } \\
\text { Negative Medium } \\
\text { Negative Small } \\
\text { Zero } \\
\text { Positive Small } \\
\text { Positive Medium } \\
\text { Positive Large } \\
\text { Positive Very Large }\end{array}$} \\
\hline & Distance Error $\left(D_{e}\right)$ & $\pm 96 \mathrm{~m}$ & & & \\
\hline Output & Throttle / Brake $(t / b)$ & \pm 1 & & & \\
\hline
\end{tabular}


TABLE III. INPUT OUTPUT LINGUISTIC RANGE

\begin{tabular}{|c|c|c|c|c|c|c|}
\hline \multirow{4}{*}{ Linguistic Values } & \multicolumn{6}{|c|}{ Range } \\
\hline & \multicolumn{4}{|c|}{ Input } & \multirow{2}{*}{\multicolumn{2}{|c|}{$\begin{array}{c}\text { Output } \\
\text { t/b }\end{array}$}} \\
\hline & \multicolumn{2}{|c|}{$\mathbf{D}_{\mathbf{e}}$} & \multicolumn{2}{|c|}{$\mathbf{V}_{\mathbf{e}}$} & & \\
\hline & $\mathbf{M}_{\mathrm{Aj}}$ & $\mathbf{S}_{\mathrm{Aj}}$ & $\mathbf{M}_{\mathbf{B j}}$ & $S_{B j}$ & $\mathbf{M}_{\mathbf{C j}}$ & $S_{C j}$ \\
\hline Negative Very Large (NVL) & -96 & \multirow{9}{*}{24} & -38 & \multirow{9}{*}{9.5} & -1 & \multirow{9}{*}{0.25} \\
\hline Negative Large (NL) & -72 & & -28.5 & & -0.75 & \\
\hline Negative Medium (NM) & -48 & & -19 & & -0.5 & \\
\hline Negative Small (NS) & -24 & & -9.5 & & 0.25 & \\
\hline Zero (Z) & 0 & & 0 & & 0 & \\
\hline Positive Small (PS) & 24 & & 9.5 & & 0.25 & \\
\hline Positive Medium (PM) & 48 & & 19 & & 0.5 & \\
\hline Positive Large (PL) & 72 & & 28.5 & & 0.75 & \\
\hline Positive Very Large (PVL) & 96 & & 38 & & 1 & \\
\hline
\end{tabular}

TABLE IV. FuZzy RULE BASE Formulated Using PSO

\begin{tabular}{|c|c|c|}
\hline \multicolumn{2}{|c|}{ Input Variables } & Output Variable \\
\hline Distance Error $\left(\boldsymbol{D}_{\boldsymbol{e}}\right)$ & Velocity Error $\left(\boldsymbol{V}_{\boldsymbol{e}}\right)$ & Throttle/Brake $(\boldsymbol{t} / \boldsymbol{b})$ \\
\hline PL & PVL & NL \\
\hline NVL & Z & NM \\
\hline NVL & NL & PL \\
\hline NVL & Z & NM \\
\hline NL & PL & PS \\
\hline PS & NL & PL \\
\hline PM & PVL & Z \\
\hline NM & PL & NL \\
\hline PL & PL & PS \\
\hline NL & PM & PM \\
\hline
\end{tabular}

TABLE V. Fuzzy Rule BASE Formulated Using DE

\begin{tabular}{|c|c|c|}
\hline \multicolumn{2}{|c|}{ Input Variables } & Output Variable \\
\hline Distance Error $\left(\boldsymbol{D}_{\boldsymbol{e}}\right)$ & Velocity Error $\left(\boldsymbol{V}_{\boldsymbol{e}}\right)$ & Throttle/Brake $(\boldsymbol{t} / \boldsymbol{b})$ \\
\hline NL & PVL & NVL \\
\hline PS & Z & NL \\
\hline PVL & PS & NM \\
\hline PM & PS & PS \\
\hline NM & NL & NM \\
\hline PM & PL & PS \\
\hline PVL & PVL & PM \\
\hline NVL & NL & NM \\
\hline NL & Z & NM \\
\hline PS & Z & Z \\
\hline Z & PL & PS \\
\hline NL & PM & NVL \\
\hline PS & PVL & NM \\
\hline PM & PS & PL \\
\hline NVL & NL & \\
\hline
\end{tabular}




\section{Testing Procedures for Performance Determination}

AEB group studied the most common crash types and analysed the same to develop and ensure test procedures for assessing the performance of FCAS system. The detailed accident analysis and test procedure are discussed in [6] and [7]

\section{A. Test Procedure Developing Organisation}

Establishments of comprehensive standards and criteria by NHTSA and standard producing organisation for assessing Forward Collision Warning System (FCWS) were dawdling [8]. NHTSA recommends testing of FCWS in three different scenarios as follows: 1) subject vehicle approaching a stopped vehicle 2) sudden deceleration of lead vehicle and 3) slower moving lead vehicle. In all these three scenarios the subject vehicle must travel at 45mph. A partial protocol has been developed by NHTSA for assessing forward collision warning system fitted in passenger cars. European New Car Assessment Programme (Euro NCAP) has developed similar test scenarios to that of NHTSA but with a wider speed range (12 to 62mph). Based on the testing procedures adopted for assessing the performance of FCWS recommended by Euro NCAP, the test procedures for assessing the performance of FCAS are developed.

\section{B. Testing Procedure, Test Track Details and Subject Vehicle Specification}

According to FCAS classification mentioned in Table I and the brake test speeds, brake testing is classified in to three major category as follows: a) Urban operating zone b) Rural Operating zone and c) Highway Operating zone. The brake test cases are categorized according to the test speeds related to the operating zones. Furthermore the status of the target / lead vehicle allows the test procedure to be categorised as a) Stationary lead vehicle, b) moving lead vehicle and c) Decelerating lead vehicle. Table VI summarises the different test scenarios considered for testing the performance. These tests are performed by creating a virtual test track using an industrial testing software CarMaker. A test track of $1000 \mathrm{~m}$ length and $7 \mathrm{~m}$ width is created. Table VII provides the specification of BMW_5 series car which is selected as subject vehicle.

TABLE VI. TEST SCENARIOS

\begin{tabular}{|c|c|c|c|}
\hline $\begin{array}{c}\text { Operating } \\
\text { Zones }\end{array}$ & Test type & $\begin{array}{l}\text { Lead Vehicle } \\
\text { Status }\end{array}$ & Description \\
\hline Urban & $\begin{array}{l}\text { CCRSs - City } \\
\text { (low speed) }\end{array}$ & \multirow{2}{*}{ Stationary } & $\begin{array}{l}\text { Subject vehicle speed is varied from } \\
10 \text { to } 40 \mathrm{~km} / \mathrm{hr} \text { in } 10 \mathrm{~km} / \mathrm{hr} \\
\text { increments }\end{array}$ \\
\hline \multirow{3}{*}{ Rural } & $\begin{array}{l}\text { CCRSs - Inter Urban } \\
\text { (low speed) }\end{array}$ & & $\begin{array}{l}\text { Subject vehicle speed is varied from } \\
50 \text { to } 80 \mathrm{~km} / \mathrm{hr} \text { in } 10 \mathrm{~km} / \mathrm{hr} \\
\text { increments }\end{array}$ \\
\hline & $\begin{array}{l}\text { CCRMs - Inter Urban } \\
\text { (initial speed @ } 50 \\
\text { km/hr) }\end{array}$ & $\begin{array}{l}\text { Decelerating from } \\
50 \mathrm{~km} / \mathrm{hr} \text { to zero }\end{array}$ & $\begin{array}{l}\text { Approaching a target vehicle with an } \\
\text { initial speed of } 50 \mathrm{~km} / \mathrm{hr}\end{array}$ \\
\hline & $\begin{array}{l}\text { CCRMs - Inter Urban } \\
\text { (low speed) }\end{array}$ & \multirow{2}{*}{ Moving at $20 \mathrm{~km} / \mathrm{hr}$} & $\begin{array}{l}\text { Subject vehicle speed is varied from } \\
50 \text { to } 70 \mathrm{~km} / \mathrm{hr} \text { in } 10 \mathrm{~km} / \mathrm{hr} \\
\text { increments }\end{array}$ \\
\hline Highway & CCRMs - Highway & & $\begin{array}{l}\text { Subject vehicle speed is set at } 80 \text { and } \\
90 \mathrm{~km} / \mathrm{hr}\end{array}$ \\
\hline
\end{tabular}

TABLE VII. SUBJECT VeHICLE SPECIFICATION

\begin{tabular}{|l|l|}
\hline Parameter & \multicolumn{1}{|c|}{ Specification } \\
\hline Vehicle Mass & $1360 \mathrm{~kg}$ \\
\hline Engine Inertia & $0.097 \mathrm{kgm}^{2}$ \\
\hline Idle Speed & $800 \mathrm{rpm}$ \\
\hline Starter Torque & $150 \mathrm{Nm}$ \\
\hline Clutch Maximum Torque & $300 \mathrm{Nm}$ \\
\hline Gear Inertia in & $1 \mathrm{e}-5 \mathrm{kgm}^{2}$ \\
\hline Gear Inertia out & $0.016 \mathrm{kgm}^{2}$ \\
\hline Synchronisation Time & $50 \mathrm{~ms}$ \\
\hline Drive & Rear \\
\hline
\end{tabular}




\section{A. Urban Operating Zone}

\section{IV.Test Results AND Discussion}

1) CCRSs City Scenario: In this test scenario (Fig. 4), the subject vehicle speed is varied from $10 \mathrm{~km} / \mathrm{hr}$ to 40 $\mathrm{km} / \mathrm{hr}$. A stationary target (lead vehicle) is kept at 900 meters away from the start line. In all the test speeds the vehicle with the proposed EFLC - FCAS controller was able to sense the stationary target and was able to bring the subject vehicle to halt avoiding rear end collision. The rest of the controllers mentioned in [1] and [2] was successful in avoiding the rear end collision (Fig 4. a to c) in test speeds except 40km/hr (Fig 4. d).

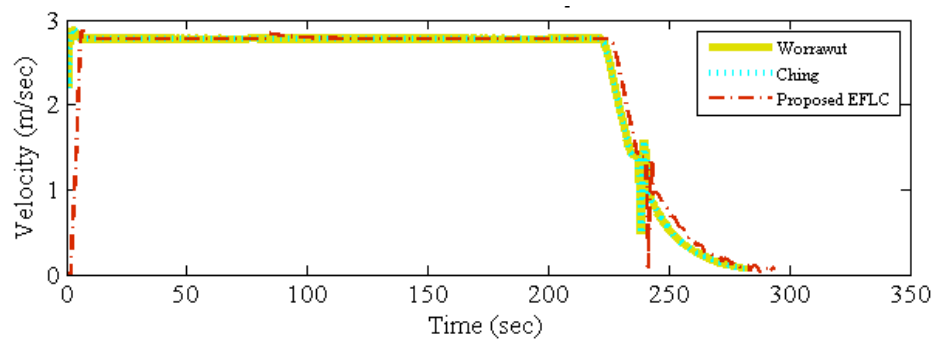

a) Test speed of $10 \mathrm{~km} / \mathrm{hr}$

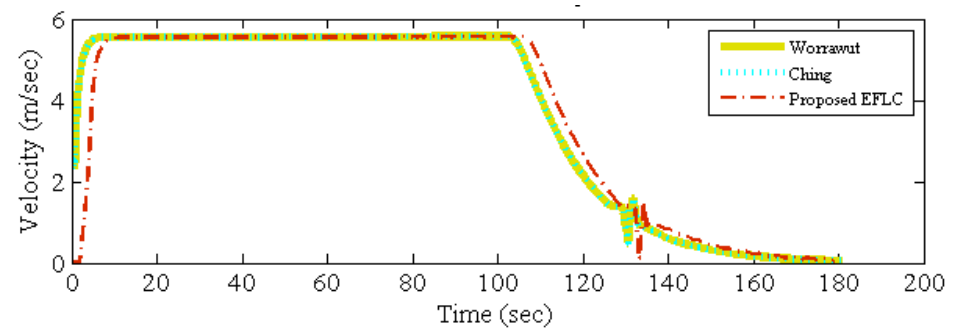

b) Test speed of $20 \mathrm{~km} / \mathrm{hr}$

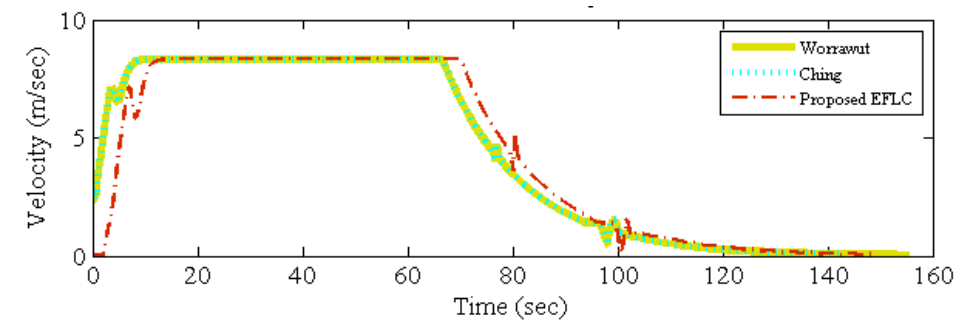

c) Test speed of $30 \mathrm{~km} / \mathrm{hr}$

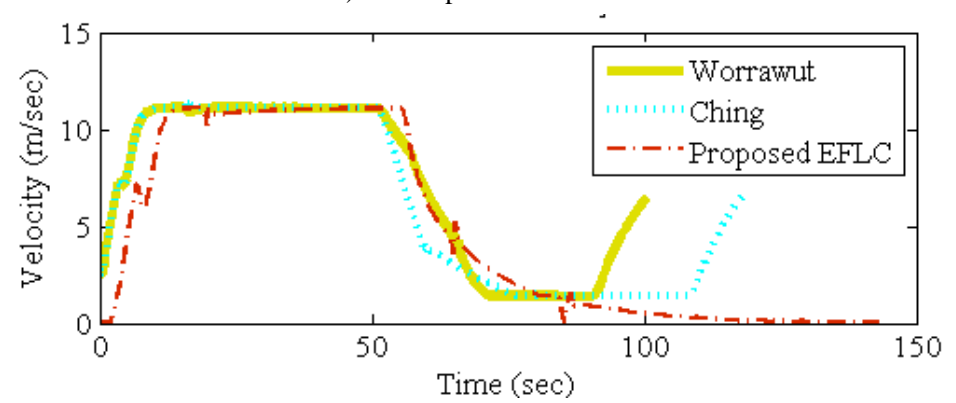

d) Test speed of $40 \mathrm{~km} / \mathrm{hr}$

Fig. 4. CCRSs - city scenario

\section{B. Rural Operating Zone}

In this operating zone the brake testing is performed in three different category. The subject vehicle is allowed to adopt to the changes of the lead vehicle when it is stationary, moving and sudden decelerating.

1) CCRSs Inter Urban (Low Speed): The subject / host vehicle is allowed to approach a stationary target / lead vehicle at test speeds between 50 and $80 \mathrm{~km} / \mathrm{hr}$. The target vehicle is kept stationary at a distance of 900meters from the start line. The test results (Fig. 5) imitate the behaviour similar to that of the CCRSs Inter Urban low speed scenario. In this brake testing scenario the proposed controller was successful in avoiding the rear end collision meanwhile the conventional controllers failed to avoid rear end collision. 


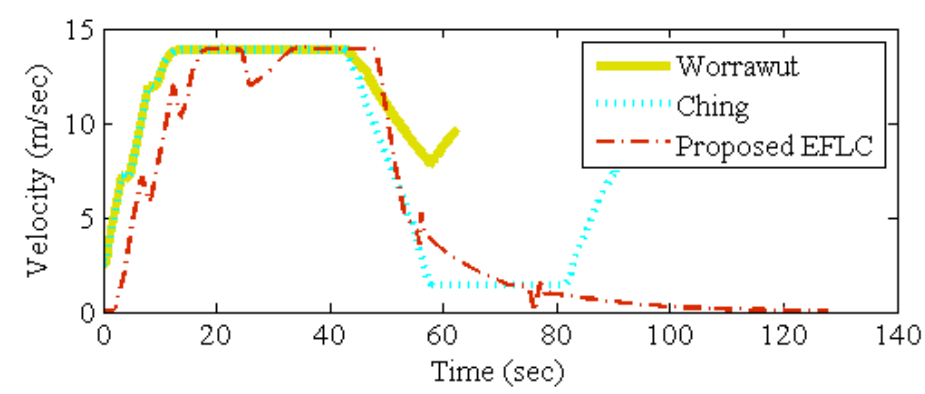

a) Test speed of $50 \mathrm{~km} / \mathrm{hr}$

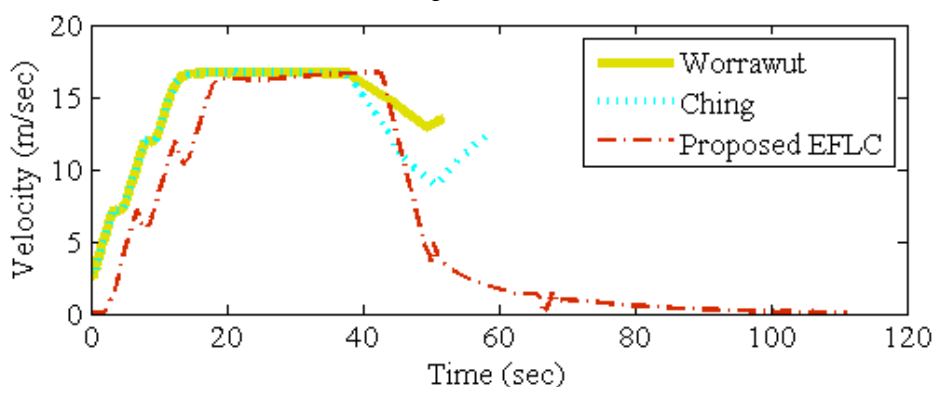

b) Test speed of $60 \mathrm{~km} / \mathrm{hr}$

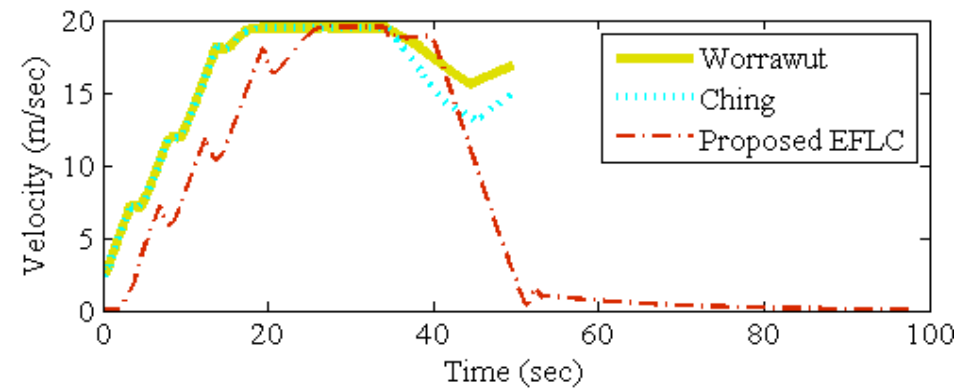

c) Test speed of $70 \mathrm{~km} / \mathrm{hr}$

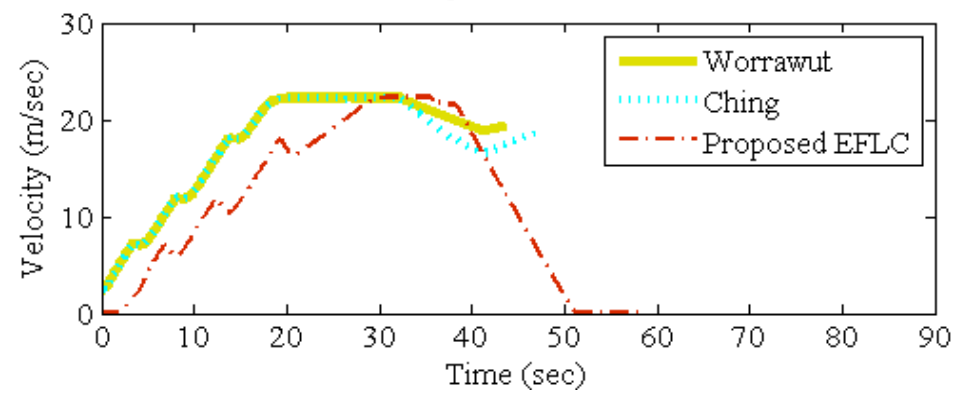

d) Test speed of $80 \mathrm{~km} / \mathrm{hr}$

Fig. 5. CCRSs - Inter urban (low speed) scenario

2) CCRMs Inter Urban (Low Speed): The test speed is between 50 to $70 \mathrm{~km} / \mathrm{hr}$ and the target / lead vehicle is allowed to move at a constant velocity of $20 \mathrm{~km} / \mathrm{hr}$. The subject / host vehicle has to reduce its speed from the corresponding test speed and avoid rear end collision. 




a) Test speed of $50 \mathrm{~km} / \mathrm{hr}$

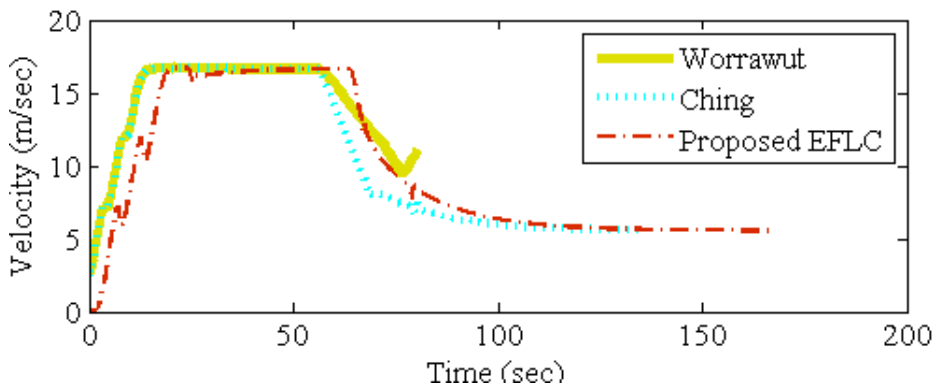

b) Subject vehicle speed at $60 \mathrm{~km} / \mathrm{hr}$

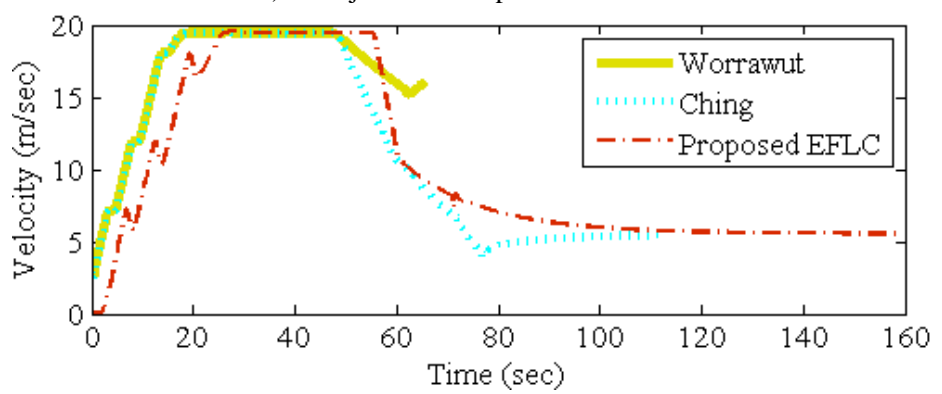

c) Test speed of $70 \mathrm{~km} / \mathrm{hr}$

Fig. 6. CCRMs - Inter urban (low speed) scenario

For all the test speeds the controller mentioned in [2] and the proposed EFLC-FCAS controller performed better over the controller mentioned in [1]. Proposed controller was able to achieve better control over all test speeds in this scenario.

3) CCRMs Inter Urban Scenario with Initial Speed of $50 \mathrm{~km} / \mathrm{hr}$ : In this brake assessment test the subject and the target vehicles are allowed to run at $50 \mathrm{~km} / \mathrm{hr}$ initially.After 50 seconds, the target vehicle decelerates suddenly to $0 \mathrm{~km} / \mathrm{hr}$.

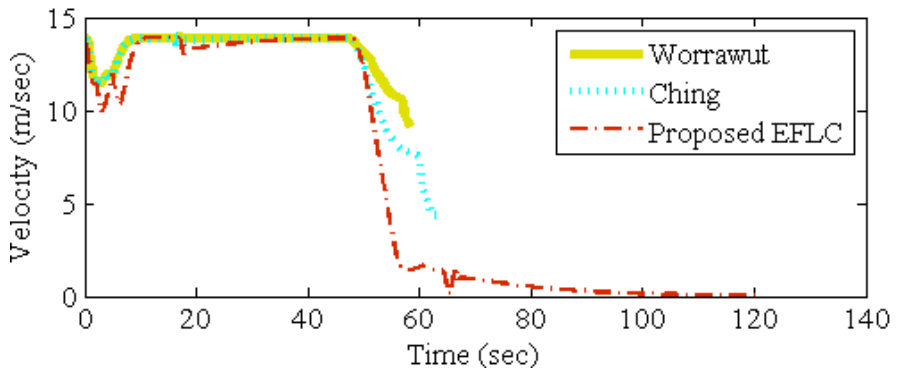

Fig.7. CCRMs - Inter urban scenario with initial velocity of $50 \mathrm{~km} / \mathrm{hr}$ for subject and lead vehicles

The proposed controller was able to bring the vehicle to zero and avoided rear end collision whereas the other two caused rear end collision (Fig. 7).

\section{Highway Operating Zone}

1) CCRMs Highway Scenario: The target vehicle (lead vehicle) is made to move at a constant speed of 20 $\mathrm{km} / \mathrm{hr}$. The test speeds were taken as $80 \mathrm{~km} / \mathrm{hr}$ and $90 \mathrm{~km} / \mathrm{hr}$. In both the test speeds, the proposed EFLC-FCAS controller alone was able to bring the subject / host vehicle to control and avoided rear end collision (Fig. 8). 


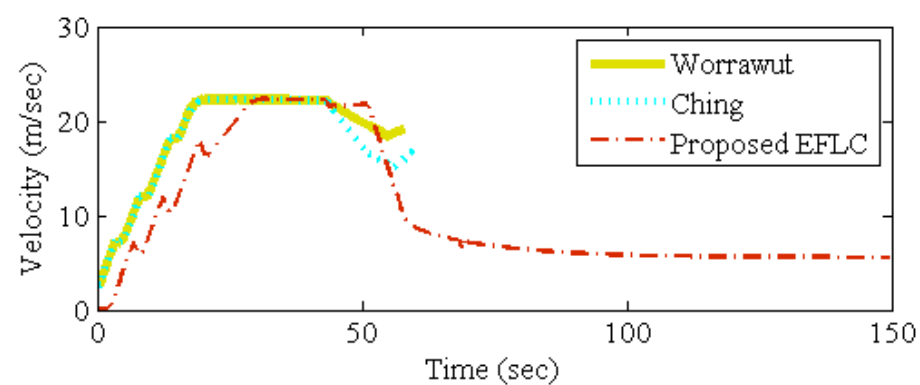

a) Test speed of $80 \mathrm{~km} / \mathrm{hr}$

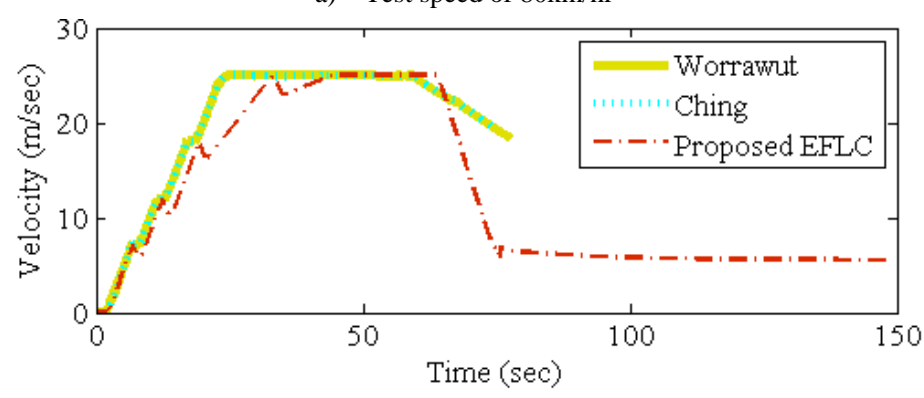

b) Test speed of $90 \mathrm{~km} / \mathrm{hr}$

Fig.8. CCRMs - Highway scenario

\section{Conclusion}

Based on the recommendations mentioned for testing forward collision warning system, the brake assessment test scenarios were developed for FCAS. These scenarios were further classified according to the operating zones. A virtual test track is created in virtual simulation software "CarMaker" and the proposed controller is created in Matlab/Simulink. The proposed controller's performance is tested and the results were compared with the similar existing controllers mentioned in earlier researches. Proposed controller exhibited a satisfactory performance by avoiding rear end collision with the lead vehicle in urban, rural and highway operating zones over its counterpart in terms of safety. Thus enabling the use of the proposed EFLC-FCAS controller in all operating zones.

\section{ACKNOWLEDGMENT}

Authors would like to acknowledge IPG, Germany for providing the trial license for CarMaker and their technical support to carry out this research work.

\section{REFERENCES}

[1] P. Worrawut, S. Thanok, and M. Parnichkun, “Adaptive cruise control for an intelligent vehicle,” 2008 IEEE Int. Conf. Robot. Biomimetics, pp. 1794-1799, 2009.

[2] T. Ching Chih, S. M. Hsieh, and C. T. Chen, "Fuzzy longitudinal controller design and experimentation for adaptive cruise control and stop\&Go,” J. Intell. Robot. Syst. Theory Appl., vol. 59, no. 2, pp. 167-189, 2010.

[3] S. P. Sathiyan, S. S. Kumar, and A. I. Selvakumar, "Optimised Fuzzy Controller for Improved Comfort level during transition in Cruise and Adaptive Cruise Control Vehicles,” in International Conference on Signal Processing And Communication Engineering Systems (SPACES-15), 2015, pp. 86-91.

[4] S. P. Sathiyan, A. W. Lins, and S. S. Kumar, “Optimization of ACC using Soft Computing Technique,” Int. J. Comput. Sci. Inf. Secur., vol. 9, no. 2, pp. 150-154, 2011.

[5] S. P. Sathiyan, S. S. Kumar, and A. I. Selvakumar, "Optimized Fuzzy Logic-Based Adaptive Cruise Control Vehicle for Urban and Highway Driving Patterns,” in Emerging Research in Computing, Information, Communication and Applications, N. R. Prasad, S. N.H., and N. Nalini, Eds. Singapore: Springer, 2016, pp. 319 -331.

[6] J. Lenard, A. Badea-Romero, and R. Danton, Typical pedestrian accident scenarios for the development of autonomous emergency braking test protocols, vol. 73. 2014.

[7] J. Lenard and R. Danton, “Accident data study in support of development of autonomous emergency braking test procedures,” 2010.

[8] “The Use of Forward Collision Avoidance Systems to Prevent and Mitigate Rear-End Crashes,” Washington, D.C., 2015. 